\title{
Guiding the Facial Rejuvenation Journey: Fulfilling the Complete Role of Surgeon and Aesthetic Practitioner
}

\author{
Mark M. Beaty, MD ${ }^{1}$ \\ ${ }^{1}$ Department of Facial Plastic Surgery, Beaty Facial Plastic Surgery, \\ Atlanta, Georgia \\ Facial Plast Surg 2021;37:140-148.
}

\begin{abstract}
Address for correspondence Mark M. Beaty, MD, Department of Facial Plastic Surgery, Beaty Facial Plastic Surgery, 950 West Peachtree St. NW, Unit 1709, Atlanta, GA 30309

(e-mail: mmbeaty60@gmail.com).
\end{abstract}
Abstract
Keywords
- facial rejuvenation
- facelift
- skin resurfacing
- volume enhancement
- facial plastic surgeon

The facial plastic surgeon's role today includes more than provision of procedural excellence. To provide excellent quality of care effective planning and guidance for patients through the aesthetic journey is needed. Methods for the delivery of this level of care are presented and discussed.

This study deals with the evolving role of a long-term, multiprocedure approach to maximize benefit from facial aesthetic procedures.

Maintenance of a youthful facial appearance is desirable and arguably even necessary in modern society for many reasons, prominently including the pursuit of social, financial, and psychological goals. We live in an age when to a greater degree than at any other time in human history powerful youth-oriented images in media coupled with an explosion of visually rich communication tools are integral parts of daily life for the general population and not just the province of celebrities, government leaders, and media figures. The pressure on our patients to maintain a vibrant and youthful appearance comes within almost all aspects of their lives, profoundly affecting their potential for success in social and business interactions. One of the most common requests we get in our practice on initial patient consultation is for help with ensuring that they look as vibrant and competent as they feel. As overall affluence in much of the northern hemisphere increases, knowledge of and focus upon healthful behaviors and lifestyle is expected to further extend the length of time many people choose to remain in the work force and active in social pursuits. ${ }^{1}$ This trend is likely to increase the demand not just for facial rejuvenation services and procedures but also for guidance in developing a strategy and plan for maintaining a youthful and imagefriendly appearance over the course of many years.

published online February 25, 2021
Issue Theme Facial Rejuvenation: A Multidimensional and Multimodal Perspective; Guest Editor: Fred G. Fedok, MD, FACS
In fact, the pursuit of physical beauty and maintaining a youthful appearance dates back at least to the dawn of recorded human history. It is no accident that legend and mythology in both western and eastern cultures are replete with ideation of some sort of fountain of youth. The Egyptians of the Middle Kingdom (ca. 2000 B.C.E.) made use of extensive beauty regimens incorporating skin care, oils and serums, cosmetics and even early attempts at surgical aesthetic enhancement and reconstruction, especially of the nose. Early attempts at aesthetic reconstruction and rejuvenation were also seen in India and the Middle East. ${ }^{2}$ Breast augmentation surgery dates back to the mid-19th century and facelifts to the late 1800s. Gradual development of aesthetic enhancement techniques continued with the surgical component taking off in the late 19th century, coinciding with the development of effective anesthesia techniques. ${ }^{3}$ Over the course of the 20th century surgical techniques have evolved to the expansive and highly technical level we know today. While initially the performance of cosmetic surgical procedures was stigmatized to the point of secrecy, ${ }^{4}$ over time sufficient interest was generated to encourage steady surgical innovation. Surgeons from disciplines including facial plastic surgery, plastic surgery, and dermatology developed surgical techniques to address reversing the common signs of facial aging including facial contour changes, volume loss, and excessive skin laxity. Over the past three decades, an explosion of technology has yielded the ability to improve appearance through energy-based (c) 2021. Thieme. All rights reserved. Thieme Medical Publishers, Inc., 333 Seventh Avenue, 18th Floor, New York, NY 10001, USA
Dol https://doi.org/ 10.1055/s-0041-1723756. ISSN 0736-6825. 
treatments, injectable products, and improved biomodulation techniques, effectively addressing many of the same concerns previously addressed only by surgery.

Over the past 20 years it has become increasingly recognized that the effective integration of a combination of surgery, volume enhancement, energy-based procedures, and biomodulation techniques, all powerful tools for aesthetic enhancement, and in particular facial rejuvenation, is a major key to getting the best outcomes for our patients. Additionally, establishing a relationship and developing an individualized care plan that includes and encourages maintenance treatments and the ongoing active participation of both patient and physician sets the stage for the most successful aesthetic enhancements. It is no longer, and actually never was, sufficient to provide a procedure or service and fail to follow-up by offering advice and assistance to our patients on how to get the most longevity and benefit from their results. The surgical or procedural result, it turns out, is one early step in the journey toward aesthetic facial rejuvenation. While powerful in their own right, procedures are best followed by enhancements from a well-defined and executed adjunctive plan focusing on fine-tuning and maintenance of result if truly outstanding results are the goal. Additional benefit from this approach is derived by retaining patients within the individual practice and motivating the patient to buy in to the complete treatment plan, including beneficial lifestyle changes. With the physician's direction and the patient's active participation in such a comprehensive treatment plan a powerful long-term aesthetic and health enhancement is much more likely.

Integrative ideas and techniques have become de rigueur in most fields of scientific and intellectual endeavor in the 21st century. ${ }^{5}$ More importantly, the evidence in aesthetic medicine supports the idea that melding techniques to address all aspects of facial aging consistently provide the best results. To be a physician leader in a modern aesthetic practice means spending the time to learn not just the procedural and medical aspects of rejuvenation techniques, but also how to support our patients in the long term and help them to maintain results. An approach incorporating attention to a patient's aesthetic needs in relation to their overall health, physically, nutritionally, and psychologically is the most responsible and effective pathway to great results and maximal satisfaction for both the patient and physician.

\section{Evolution of Surgical Options}

Modern facial rejuvenation techniques began to evolve in the 1960s when the first consistent advocacy for modifying supportive structures of the face began to emerge. While attempts at surgical reconstruction and aesthetic enhancement are described in both the European and U.S. literature in the early 20th century, these techniques were essentially limited to skin excision. ${ }^{6,7}$ The history of modern facial rejuvenation surgery slowly progresses from around the end of World War I as two concurrent developments emerged. First, demand for reconstructive surgery after the two World Wars fueled the learning curve for facial procedures and surgeons learned to adapt reconstructive techniques for rejuvenative purposes. Second, safer and more effective anesthesia became a commonplace, making all types of elective surgery, cosmetic surgery included, much more practical. Still, the majority of techniques described during the first half of the 20th century were some variation of skin only excision and tightening, providing improvement in skin laxity but little long-term change in facial contour. Description of a technique by Skoog advocating incorporation of the platysma muscle and associated fascia stimulated interest in incorporating supportive tissue of the face for rejuvenation. ${ }^{8}$ Subsequent description of the superficial musculo-aponeurotic system (SMAS) layer by Mitz and Peyronie and development of techniques for restoring the position and integrity of this critical supportive tissue ushered in the era of effective long-lasting facelifting and made possible modern facial rejuvenation. ${ }^{9}$ Many varieties of SMAS manipulation are described ranging from suture plication to excision of segments of SMAS with limited elevation and imbrication to more extensive sub-SMAS dissection and separate elevation and fixation of this tissue with subsequent redraping of skin, potentially allowing multiple vectors of correction to be used. $^{10-12}$

SMAS techniques remained limited in the ability to improve the midface, nasolabial folds, and jowls, however. To mobilize the midfacial tissue and maximize improvement over the malar eminence and at the nasolabial fold, mobilization of a thicker composite flap dissected beneath the SMAS was advocated by Hamra and popularized as the deep plane technique. ${ }^{13}$ Other authors expanded upon this idea with emphasis on release of retaining ligaments at the zygomatico-cutaneous and mandibulo-cutaneous fixation points. Complete release of these areas allows much greater mobility of the supportive flap as well as wider choice of vector for advancement. Proponents of these approaches argue that in addition to better correction at the midface and jowl, these techniques provide longer-lasting correction and a more natural appearing result. ${ }^{14-16}$ Combining the potential advantages of each of these techniques, Beaty described a systematic approach to individualizing the deep tissue technique based upon the progressive evaluation intraoperatively of each particular patients anatomy and needs, then utilizing the combination of techniques best suited for that individual. ${ }^{17}$

\section{Recognition of the Role of Volume}

\section{Deep Plane Facelift}

As previously discussed, one of the difficulties encountered with various SMAS lifting techniques is failure to achieve adequate correction in the midface which generally suffers from both volume loss and volume descent. Hamra popularized the creation of a composite flap elevated in a sub-SMAS plane inferolaterally and then transitioned over the mimetic musculature at the zygomaticus major. ${ }^{18}$ The deep plane facelift has enjoyed great popularity with refinements proposed by many authors. Emphasis on full release of the zygomatico-cutaneous ligament has been a key feature in maximizing improvement in the midface by vertical advancement of the ptotic malar tissue. Recent refinements by Jacono ${ }^{14}$ advocating greater midface 
elevation and mandibular ligament release and by Gordon and Sawan ${ }^{16}$ advocating concurrent modification of the buccal fat pad may provide even greater contouring and improvement. One of the keys to understanding the power of these techniques is their ability to allow effective repositioning of descended malar, buccal, and jowl fat pads, in many cases restoring adequate volume to contours lost to both aging-associated volume loss and volume descent.

\section{Fillers}

The development of effective, long-lasting fillers significantly improved the ability of facial plastic surgeons to reverse the signs of aging. Over the past 15 years there is greater recognition amongst surgeons that deflation of natural volume in the face significantly contributes to the stigmata of aging accentuating loss of natural contours and soft tissue descent. ${ }^{19}$ Particularly in the female face loss of width at the midface coupled with descent of the lower cheek tissue tends to yield a more-square shape to the face, masculinizing its appearance. In the early 1990s collagen began to be replaced by longer lasting and safer fillers including hyaluronic acids, calcium hydroxyapatite, and L-polylactic acid. The great advance provided by these materials was the ability to achieve smooth and natural-appearing effects lasting 6 months or longer, finally providing an option for facial plastic surgeons to conveniently and economically manage mild to moderate volume loss as a component of facial aging. Additionally, the emerging popularity of procedures such as lip augmentation was significantly advanced by the availability of these products as they made low down time office procedures possible. There continues to be a significant limitation to the use of off the shelf injectable fillers, however, which is the ultimate metabolization of the respective fillers and consequently the requirement for regular reinjection and the cost associated with correcting greater degrees of volume loss in patients with more advanced facial aging.

\section{Fat Transfer}

Fortunately, techniques of fat transfer which were developed and improved over a similar time period have proven to be an excellent option for long-term correction of facial volume deficits. Especially for patients with more advanced aging changes, fat transfer offers a more resource effective treatment due to its durability and relatively lower cost for larger volume correction. Initially popularized by Coleman, ${ }^{20}$ fat transfer is now developed into a multifaceted technique encompassing not just volume augmentation but use of specialized autologous fat products such as microfat and nanofat which primarily work by contributing growth factors from fat cells to augmented tissue. With experience in processing autologous fat, it is generally possible to reliably achieve $90 \%$ survival of transferred fat volume in the face allowing for excellent long-term correction that some surgeons incorporate into their facelift. Others choose to use fat augmentation for additional fine-tuning after a primary facelift procedure or as a standalone treatment when volume loss is a primary issue for a particular patient.

\section{Importance of Technology-Based Solutions}

\section{Laser}

Laser resurfacing technology continues to improve and has now largely, if not completely, supplanted chemical peeling for moderate to aggressive skin resurfacing. It is well-recognized that skin surface rhytids, pigment irregularities, and sun damage are significant contributors to the stigmata of facial aging. Even with excellent contour correction from advanced facelifting techniques, wrinkled and sun-damaged skin in the aging patient retains an aged appearance. Patients seeking to maximize improvement in aging face changes almost always benefit from skin resurfacing techniques in some capacity. It is rare that the facial plastic surgeon sees significant changes in facial contour without at least some degree of surface lines and wrinkles or texture change. Resurfacing techniques therefore are an important part of complete facial rejuvenation. For those living in warmer climates or who are active outdoors the additional problems of solar keratosis and pigment irregularities may also be dramatically improved with skin resurfacing methods.

\section{Ultrasound}

Microfocused ultrasound was introduced in 2009 as an option for improving facial contours without surgery and can serve as a useful stand-alone treatment for patients with early sagging of deep facial tissues. There is considerable controversy about the efficacy of these treatments with more current evidence suggesting that with proper protocols and density of treatment pattern significant improvement in brow, lower facial, and neck contours are possible. MIcrofocused ultrasound offers a useful option for patients with mild facial aging changes who may be able to achieve their goals without surgery, avoiding downtime and limiting short-term costs. There can also be a role for this technology in patients who cannot consider elective surgery because of underlying medical conditions.

\section{Radiofrequency}

Radiofrequency technologies were introduced in the early 2000s and were initially limited to broad field, monopolar skin surface treatments. Initial issues with variable efficacy and patient discomfort during treatment were gradually improved with each new generation of devices. The most popular radiofrequency devices currently are bipolar or multipolar minimally invasive devices using microneedle delivery systems. Radiofrequency energy is passed between the needles creating well-controlled tissue heating which in turn stimulates production of new skin components including collagen, hyaluronic acid, and in some cases, elastin. These devices have proven especially useful for skin tightening and improvement of surface texture and have the advantage of being office-based requiring minimal anesthesia and with minimal downtime.

\section{Effective Maintenance Procedures}

Once improvements in facial contour, volume restoration, and skin surface are achieved, most modern patients are 
interested in the best ways to maintain or even enhance results. Patients are increasingly used to participating in longer term health maintenance as the last decade has seen an increase in emphasis on health and wellness programs organized through employers, social clubs, and fitness organizations. Fortunately, methods for maintaining results, especially for skin have improved greatly and now offer great promise for extending the benefits of facial aesthetic surgery.

\section{Ultrasound}

MIcrofocused ultrasound can be a powerful tool for maintenance treatment in many patients. Frequently patients may have concerns with small facial contour changes a few years out from a major surgical procedure which do not merit consideration of further surgery. When these concerns are related to areas of deep tissue support such as the lower cheek and jowls, mild neck laxity or brow ptosis, this modality can provide sufficient tissue tightening to restore facial contours to the level obtained following surgery without downtime or further invasive procedures. Treatment cost is substantially lower and as with its use in early facial aging changes, microfocused ultrasound used periodically on an ongoing basis may significantly delay the need for any further surgical intervention.

\section{Microneedling}

Microneedling has become increasingly popular for maintenance of skin texture, tone, and quality and has proven to be a very effective technique for improving some early aging changes in skin tone and texture. Especially when combined with nutrient serums and growth factors the improvement can be dramatic, especially when regularly performed. In comparison to earlier techniques for minimally to noninvasive skin therapy such as microdermabrasion the results are significantly better. Presumably, the direct stimulation into the dermis from the repeated insertion of microneedles promotes vigorous collagen regeneration. The micropunctures also offer a route of ingress to nutrient serums and growth factors, increasing their efficacy dramatically over topical application. Additionally, in many locales these procedures can be delegated under supervision to aestheticians or medical assistants, making the modality more highly accessible to patients.

\section{PRP}

Interest in biomodulators including platelet rich plasma, nanofat, and exosomes is growing. While reviewed and confirmed clinical evidence for efficacy remains in the early stages, there appears to be promise of meaningful results in stimulating rejuvenation of skin and soft tissue. Application in other fields, especially orthopaedics, has also been promising, further supporting the view that as we learn more about these products, they will play an increasingly important role in facial rejuvenation efforts. Since many of these products are autologous substances there is a little risk of adverse reaction. So, safely issues are limited to harvest and injection technique.

\section{Skin Care and Wellness}

\section{Topicals}

Our dermatology colleagues have long recognized the utility of topical treatments for improving skin quality. While the treatment of unusual or complex problems in skin care may be beyond the scope of facial plastic surgeons to treat, many of our patients benefit from routine design and use of a skin care regimen including topical chemoexfoliants, especially the retinoids, and treatments for inflammatory problems such as acne rosacea which may include topical antibiotics and antiinflammatories. Like many of the other treatments outlined here topical medications are often most effective when combined with other therapies designed to improve skin quality including IPL and other energy-based technologies.

\section{Medical Grade Products and Guidance}

Patients seeking the best outcomes from facial rejuvenation also benefit from using higher grade daily skin care products than those frequently available over the counter at retail cosmetic outlets. The basics of top-quality skin care should always include cleansing, exfoliation, hydration and moisture protection and sunscreen. There are several excellent medical grade product lines available for distribution and recommendation from medical offices. For physicians who wish to more ambitiously pursue product use by their patients, development of private label or even custom formulation of product lines is available.

\section{Promoting General Health}

In addition to the improvements we make to our patients' appearance as surgeons, it is well recognized and should be patently obvious that promoting good general health results in better healing, delayed development of the signs of aging, and better maintenance of long-term results. Furthermore, as physicians we should be champions of overall health for our patients as an integral part of our treatment plan no matter what specialty we practice. Despite our position as super specialists in facial plastic surgery, we are physicians first and have all pledged to place the overall health and wellbeing of those under our care as first priority. The question then becomes how can we effectively offer health promotion within our practices or as a more convenient adjunct by associating with other providers in either referral relationships or direct incorporation of a medical component to our aesthetic practices.

\section{Putting It All Together}

\section{How We Do It within Our Practice}

The preceding overview of available techniques and technology for the practicing facial plastic surgeon outlines the scope of possibilities for getting and maintaining outstanding results for our patients. While at first blush the scale and scope of available treatments may seem daunting to incorporate into the confines of a single practice, I have come to believe that a broader range of offerings to patients allows facial plastic surgeons an unprecedented opportunity to help people more effectively 
meet their aesthetic goals. When done effectively with adequate focus and training for involved staff members, offering a comprehensive suite of services establishes the practice as not only a leader in innovation, but as an organization that places the overall well-being of its patients at the forefront. As part of this process, however, facial plastic surgeons and their support team must dedicate significant time and resources toward effectively shifting their focus to a more broadly encompassing mindset, with less emphasis on promoting specific surgeries or treatments and rather emphasizing the long-term relationship between physician, practice, and patient intended to foster a consistently and persistently outstanding result.

The starting point for successfully providing such full scope guidance to patients is deciding how many of the services required may be successfully offered within the practice. There is no universally correct answer for this question as the decision-making process involves many factors. The experience level and interest level of the physician as the team leader is the first consideration to be taken in deciding how many services to offer directly within the practice. In our experience, the practice started focusing almost entirely on surgery as these services were predicated on the smallest number of providers (myself) and the least additional capital expense. For the first several years of practice this approach served us well allowing a relatively small staff to provide excellent service with low overhead. Many adjunctive procedures using energy-based technology and those focusing on skin care or maintenance therapy were outsourced, which had the additional benefit of establishing relationships with other practices and businesses who became reliable referral sources. As the surgical reputation grew, we found that many of our patients expressed interest to us in having a more extensive palette of services available in our location as a trusted source of care and for added convenience. A critical decision point was to determine that it would not only be possible but advisable to expand our scope of care into emerging energy-based technologies.

How one incorporates additional service lines into a practice is the next step in developing the provision of a patient experience of comprehensive guidance through the entire aesthetic journey. Many of the device-based procedures, especially those using new or novel equipment, are initially quite expensive for capital acquisition requiring an expanding practice to either divert significant income or take on debt risk to venture into these areas. Other types of service addition, such as injectables, fat transfer, and biomodulators have a much lower cost of entry, primarily related to the cost of materials, without a need for large capital expenditures. This lower cost of entry makes these service lines a more common initial addition to most practices. The lower barrier to entry may engender greater competition, however, at least in some markets. Especially since recognizing the importance of volume augmentation for fully effective facial rejuvenation our practice has been aggressive in adding a wide variety of injectables services. All are directed by physician-established protocols though we offer several different styles of service. Physician injections are geared primarily toward achieving a desired effect and are consulted and priced accordingly while mid-level and nurse injectors offer a more traditional by the unit or by the syringe set of services. This allows a great deal of fine tuning of both price point and amount and frequency of product used depending on patient needs and preferences. In addition, we have found fat transfer to be a very useful component of addressing the aging face in many patients who have experienced greater degrees of volume loss. In this setting, it actually becomes more cost efficient to use fat transfer than the large volume of fillers at significant cost per syringe. As will be seen in patient case examples, some patients can remain stable for long periods of time from a facial appearance standpoint with little more than use of neurotoxins and fillers coupled with high quality skin care.

Technology-based solutions can be a powerful adjunct to surgery and injectables, adding effective ways to tighten and regenerate supportive tissues and skin. Incorporation into a practice can be costly, yet it should be recognized there are an increasing number of avenues to reduce upfront investment for the practice including the purchase of used equipment, short-term leasing options, and an increasing number of companies offering daily or per case rental arrangements for this type of equipment. Most patients seeking facial rejuvenation who have reached a moderate to advanced stage of facial aging change have at least some degree of skin surface changes with or without skin laxity. Most of these aspects of facial aging are treatable with laser or RF technologies so the addition of well-selected devices for treatment of these problems can be a tremendous benefit to patients in their overall pursuit of facial rejuvenation. In our practice technology acquisition was accomplished on a gradual basis with much thought given to the integration of each device. Five criteria were met before bringing a device into the practice: (1) It must provide a service or result that our patients want and ask for; (2) It must be effective in accomplishing the promised result on a consistent basis; (3) It must be safe to use with minimal risk of complications; (4) It must be a reliable device with minimal history of breakdown and rapid service support in the event of any problems; (5) It must provide a good value for the patient as well as an acceptable return for the practice. Only when these criteria are met do we consider acquisition of a piece of equipment and begin the decision process for how to purchase, lease or rent the particular device.

Over the course of time, we can see, it is possible for a facial plastic surgery practice to effectively make a wide variety of services available to patients. The advantages of this more expansive approach to aesthetic practice are multiple and when used by the directing physician to create a plan that truly guides the entirety of the facial rejuvenation journey from primary procedures through secondary and supportive procedures and a long-term maintenance plan results and satisfaction will improve. Not only will patients obtain a better result, but they are more likely to have appropriate expectations when a complete and extended plan is presented to them. There is a higher likelihood of buy in from the patient, increasing motivation to follow-up with longer term recommendations such as skin care and regular 

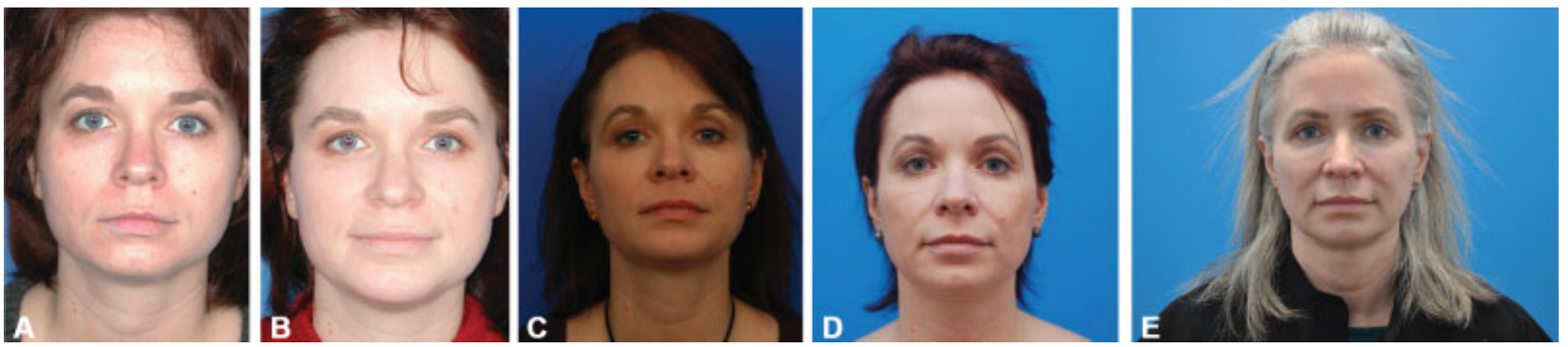

Fig. 1 Beaty: guiding the facial rejuvenation journey: (A) 2000, (B) 2005, (C) 2010, (D) 2015, (E) 2020.

use of maintenance treatments such as injectables, microneedling, and IPL treatments. Relationships are strengthened between the patient and practice staff, building trust and loyalty on both sides. This not only helps to increase the number of services provided by the practice but also tends to make referrals to others for services the practice does not provide stronger and more effective. Our practice has certainly experienced better results, longer average patient relationships, and increased satisfaction as we have become more adept at seeing and marketing ourselves as a comprehensive source for our patients' aesthetic needs and not just a provider of a particular service.

\section{Patient Case Examples}

The proof of the merit behind this practice philosophy is the long-term results we are able to offer to our existing and prospective patients. Our practice was never satisfied with the number of patients who discussed with me during consultation their disappointment and frustration with procedures they had obtained prior to seeking us out, not because the result was not good, but rather because they found themselves some years later lacking the maintained improvement in appearance they had hoped for. We present patient examples showing the power of integrated techniques to maintain a rejuvenated appearance over time along with a brief description of how this was achieved in each case.

Patient example number 1 began treatment with our practice in 2000 at age 30 . Her goals were to maintain a youthful yet natural appearance and to be able to frequently go without makeup as her busy schedule often made daily appearance preparation impractical. When initially seen she also wanted to enhance the appearance of her chin and neckline. After the initial consultation we began with a medium anatomical chin implant and a midline platysmaplasty. We also initiated a skin care program and regular use of neurotoxin for upper facial wrinkle control. These methods provided good control for her until 2008 when she began to notice some sagging of the facial tissues and accelerated soft tissue volume loss. We added occasional use of injectable fillers to her regimen as well as nonablative laser treatments for skin tightening. In 2010, she became concerned with redundant skin on her upper eyelids and pigment and texture changes of the facial skin. We performed an upper eyelid blepharoplasty and a full-face nitrogen plasma resurfacing procedure. This patient did very well and remained satisfied with her facial appearance for several years with just periodic neurotoxin use. In 2015 she became more concerned with facial contour changes including early jowl formation and relaxation of the brow. At this time, we elected to proceed with microfocused ultrasound for improvement of supportive tissue laxity and radiofrequency augmented microneedling for skin tightening. We also performed a medium depth chemical peel. From 2015 to 2017 this patient had maintenance treatments with neurotoxins, fillers, and conventional microneedling. In 2017 she noticed further progress of her facial contour changes and we elected to proceed with a limited incision facelift. Subsequently, the patient had maintenance skin treatments with IPL and Erbium YAG laser in addition to continuing regular use of neurotoxins. Most recently, in 2020, she underwent a revision upper lid blepharoplasty in the office and full-face Ulthera treatment. The series of photos shows her progression from 2000 to present (-Figs. $\mathbf{1}$ and $\mathbf{2}$ ).

The second patient presented to us requesting facial rejuvenation in 2005 at age 55 . She had a long history of sun exposure and spent a significant amount of time outdoors. Her aging changes were significant with loss of support in the lower face, midface and neck, redundant
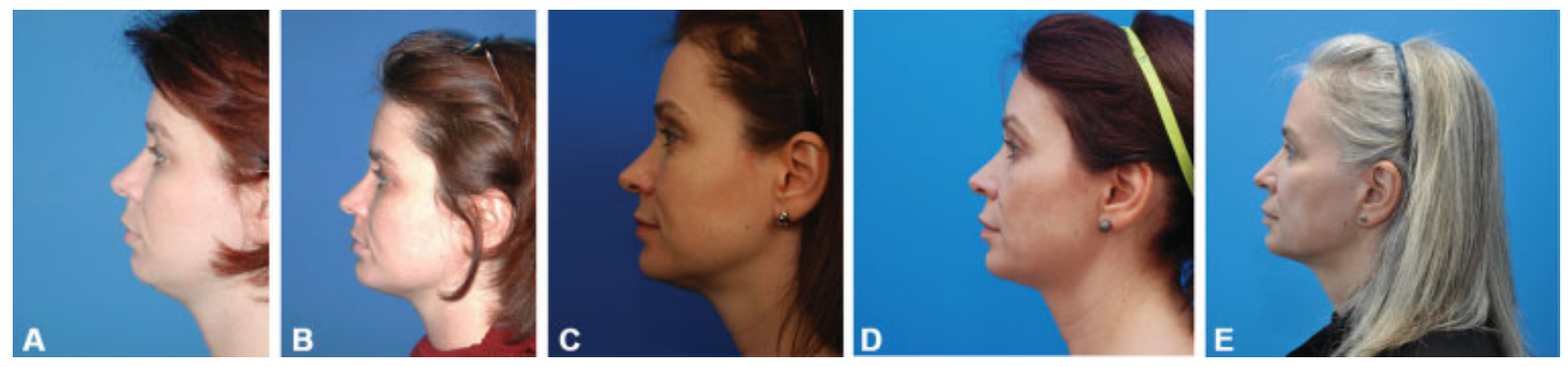

Fig. 2 Beaty: guiding the facial rejuvenation journey: (A) 2000, (B) 2005, (C) 2010, (D) 2015, (E) 2020. 

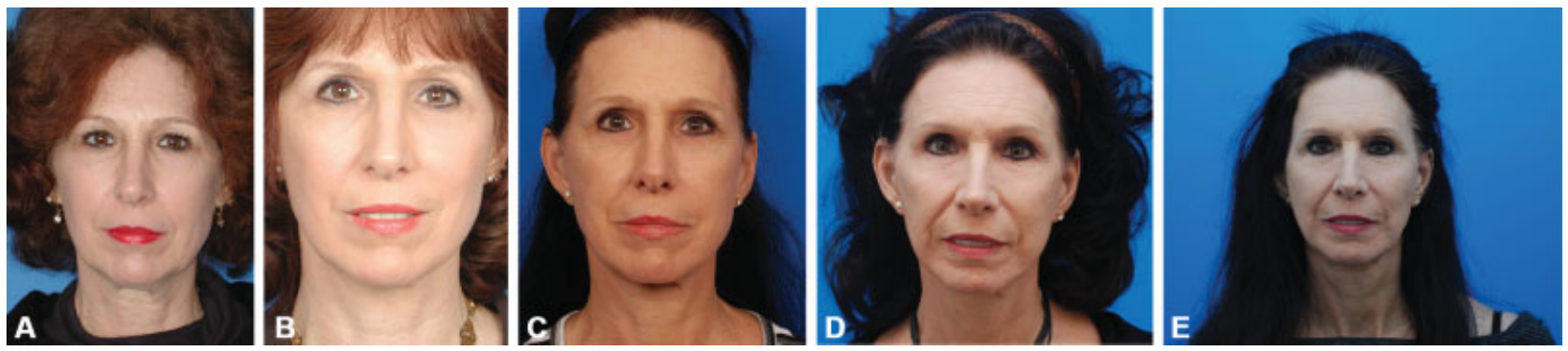

Fig. 3 Beaty: guiding the facial rejuvenation journey: (A) 2003, (B) 2005, (C) 2012, (D) 2015, (E) 2020.

tissue of the eyelids and significant sun damage and loss of skin elasticity. We began with a comprehensive surgical facial rejuvenation including endoscopic browlift, upper and lower eyelid surgery, face and neck lift. We also performed a medium depth chemical peel 2 days following her surgical procedures. Excellent correction was achieved, and we started this patient on a directed skin care regimen. She was already nutrition-conscious, and we added a greater focus on sunscreen as she participated in many outdoor activities. We developed a plan for her to begin maintenance therapy in an effort to prolong her surgical results. This patient was not comfortable with the idea of neurotoxins, so we focused on skin care and fillers. In addition to regular use of fillers we performed a full face Ulthera treatment in 2008 and in 2012. These efforts yielded excellent maintenance of results and the patient was very happy with her progress. I saw her to adjust her plan at least annually. In 2015 there had been sufficient progress of her aging face changes that we decided upon an additional major intervention which included a second facelift and browlift. At an interval of 2 months we performed a full-face chemical peel. In 2016 the patient felt she had excessive redundant skin of her upper eyelids so we added and upper eyelid blepharoplasty. Most recently, due to accelerated volume loss in the face we elected to perform an in-office fat transfer procedure rather than continue with hyaluronic acid fillers. The series of photographs shows the progress of this patient from 2005 to 2019 (-Figs. 3 and 4).

The third patient began seeing us for nonsurgical office procedures in 2012 at age 53. She was most concerned with changes to her skin, especially fine lines and wrinkles which were effectively managed with neurotoxin and fillers. This provided effective management of her facial aging for several years. In 2015 the patient felt that her lower face and neck changes had progressed to the point she wanted surgical intervention. We performed a limited incision facelift with good results. One year later we also performed an upper eyelid blepharoplasty in the office. After these procedures this patient did very well with maintenance primarily consisting of fillers and neurotoxins. In 2019 she had an unfortunate accident causing significant lacerations to her lip and chin which were effectively repaired with geometric broken line closure. The series of photographs demonstrates her progress with our practice from 2012 to present (-Figs. 5 and $\mathbf{6}$ ).

\section{Conclusion: Why I Believe in What We Do}

Facial plastic surgeons can and should see themselves as an advocate and guide for the aesthetic journey of each patient. It is no longer sufficient to be an excellent surgeon or injector or device expert and expect to meet the needs and desires of the sophisticated patients that comprise today's market. While each individual procedure plays an important role in the overall result a patient may achieve, the combined effect of a well-planned series of treatment interventions will often yield a result even better than the sum of its parts. There are three major components which contribute to the typical appearance of the aging face: (1) position and laxity of the soft tissue supportive structure of the face, (2) changes in facial volume which may include soft tissue changes, and (3) changes in the underlying bony framework of the face, and skin elasticity, tone and texture. Addressing each of these components in a comprehensive and effective manner is necessary to obtain the best possible results in facial rejuvenation. Thus, the facial plastic surgeon is best served by guiding each patient, personally or as part of a multilevel team, through the options
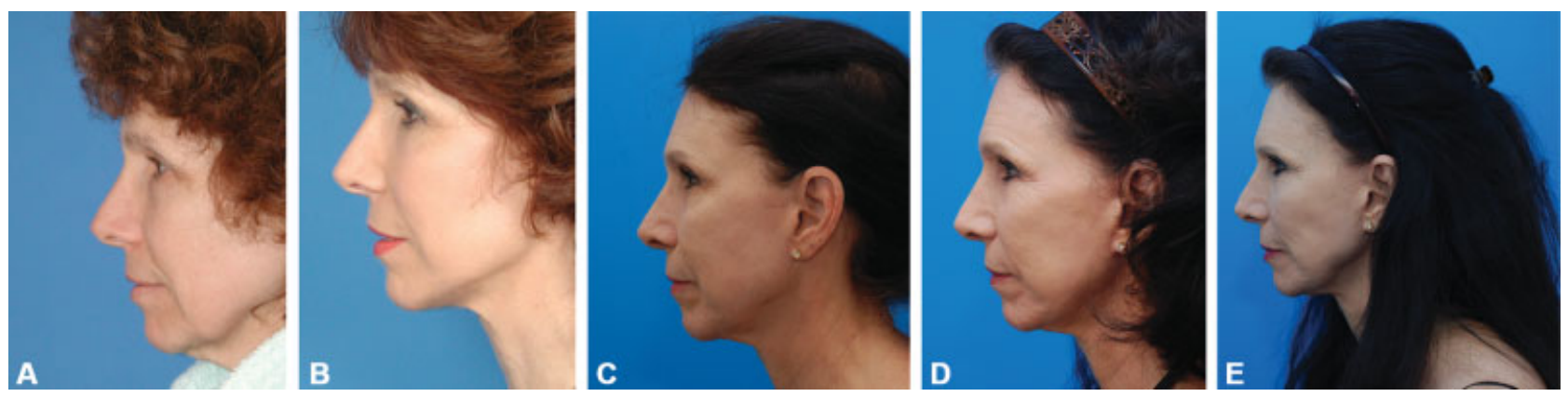

Fig. 4 Beaty: guiding the facial rejuvenation journey: (A) 2003, (B) 2005, (C) 2012, (D) 2015, (E) 2020. 

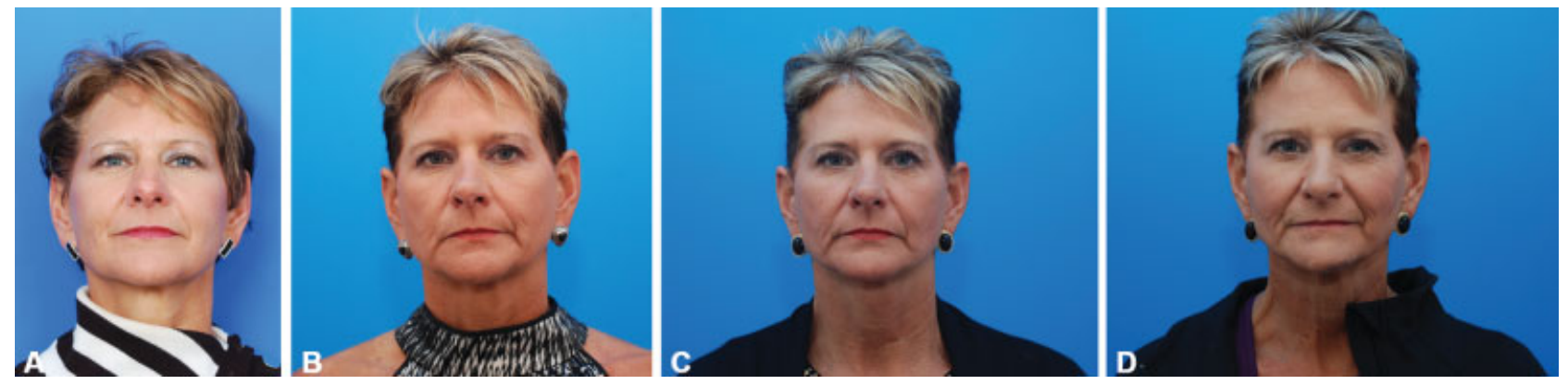

Fig. 5 Beaty: guiding the facial rejuvenation journey: (A) 2009, (B) 2015, (C) 2017, (D) 2019.
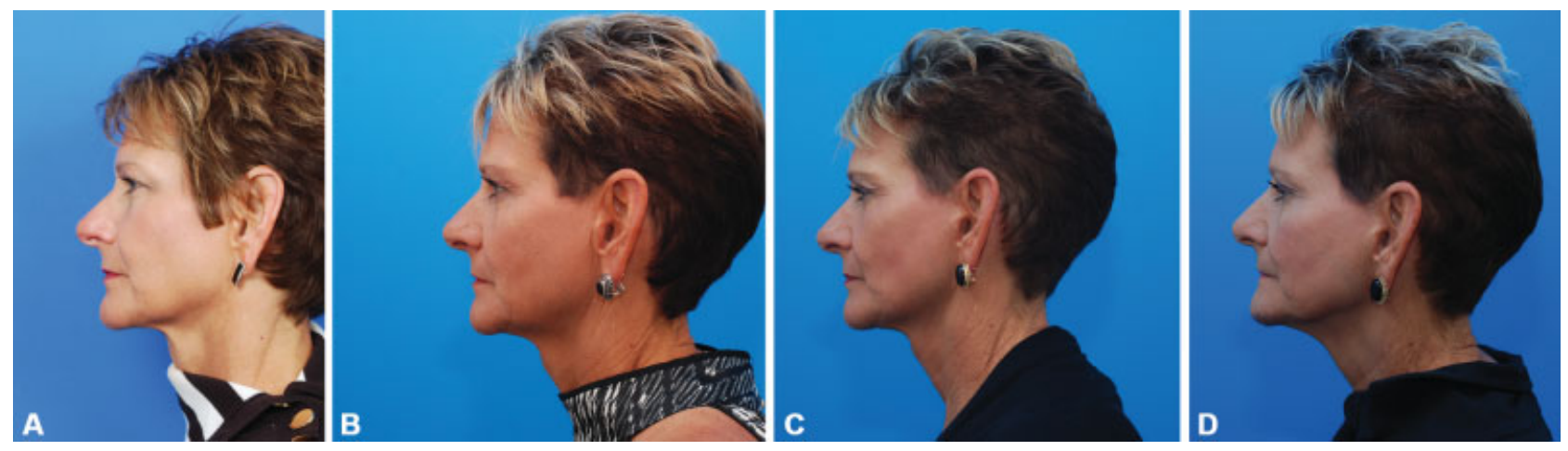

Fig. 6 Beaty: guiding the facial rejuvenation journey: (A) 2009, (B) 2015, (C) 2017, (D) 2019.

suited to them for correction and maintenance of each of that patient's primary facial issues.

Advances in surgical technique from the 1960s to the present offer the ability to dramatically improve the appearance of the aging face while maintaining natural appearance and minimizing any visible incisions. Particularly since the advent of facelifting techniques which more effectively improve facial contour while also allowing greater flexibility and customization according to the needs of the patient, facial rejuvenation surgery has become more powerful and popular than ever before. This powerful fundamental tool of facial rejuvenation is often the centerpiece of efforts to minimize the appearance changes associated with aging. It is an added bonus that more advanced deep plane and progressive approach techniques also yield longer-lasting results.

The temptation of rest solely on surgical technique, however, would sell our patients short as the increased recognition of the importance of proper volume restoration for improving the contours of the face has proven near equally important. While many of the techniques allowing more complete release of supportive facial structures allow better repositioning of volume descent than older techniques, especially in the midface, there are a large number of aging face patients who have a concurrent large volume loss in the midface. If this is left uncorrected the ultimate result will be less than ideal. Familiarity with the scope of volume enhancement techniques including injectable fillers, fat transfer, and facial implants is critical to uniformly obtain the best possible results.

Technology improvements make modern skin resurfacing a powerful component of total facial rejuvenation, especially when combined with advanced surgical approaches such as deep plane facelift techniques and ProLIFT technique along with volume restoration. A wide variety of energy-based techniques including laser, radiofrequency, plasma, and microfocused ultrasound are available to effectively treat multiple skin types. These technologies further enhance the improvements provided by surgical and volume enhancing procedures by maximizing skin quality and elasticity while reducing lines, wrinkles, and sun damage.

Once the foundation is laid, the utility of maintenance treatments including neurotoxins, fillers, PRP, and in office procedures such as microneedling, RF microneedling, and nonablative laser becomes ever more effective for the long-term maintenance of result patients are seeking. Patients understand the value of long-term guidance and planning for making the most of their aesthetic efforts and creating the most value for resources used. Our practice has found that patients welcome an offering of partnership to help them with continuing care as well as initial procedures. Adding health and nutrition promotion to each patient's plan along with effective guided skin care and an emphasis on sun protection yields even greater stability of results and improvement of rejuvenate effect.

The facial plastic surgeon's role today has evolved to encompass not just procedural excellence but also to serve as the director of a team charged with developing a relationship with each patient, determining their goals, and bringing all needed resources to bear on meeting those goals. Providing guidance and encouragement as each patient progresses through this journey is as critical for superior patient results as surgical skill and technological knowledge. To be effective, at various times along the way the facial plastic surgeon must be a psychologist, 
coach, cheerleader, and an advocate. To this end, continuing education in the design and management of ongoing aesthetic strategies and best practices for maintenance therapies are important additions to the technical proficiency of the surgeon. Each practice will be different in the precise scope and scale of services offered inhouse but all should be actively participating in planning and advice to their patients regarding best outcomes, even when some of the needed services are referred out. Our patients deserve nothing less. Only when we arrive at the ability to routinely accomplish all of these things for our patients do we realize the full potential of modern facial rejuvenation.

\section{Conflict of Interest}

None declared.

\section{References}

1 Brassfield M. Beauty and Salary: How Does Employee Attractiveness Affect Lifetime Pay? Payscale. 2012

2 Newton J. A history lesson about the origins of plastic surgery. Australasian Soc Aesth Plas Surg 2018

3 Haiken E. The making of the modern face: cosmetic surgery. Soc Res (New York) 2000;67(01):81-97

4 Ryan RFA. A 1927 view of cosmetic surgery. Plast Reconstr Surg 2000;106(05):1211

5 Wake $\mathrm{MH}$. Integrative biology: science for the 21st century. Bioscience 2008;58(04):349-353

6 Hollander E. Diekosmetischechirurgie. In: Joseph M., ed. Handbuch der kosmetik. Leipzig: Verlag von Viet; 1912

7 Miller C. The Correction of Featural Imperfections. Chicago: Oak Printing; 1907
8 Skoog TWB. Plastic Surgery: New Methods and RefinementsPhiladelphia, PA: Saunders1974

9 Mitz V, Peyronie M. The superficial musculo-aponeurotic system (SMAS) in the parotid and cheek area. Plast Reconstr Surg 1976;58 (01):80-88

10 Baker DC. Lateral SMASectomy. Plast Reconstr Surg 1997;100 (02):509-513

11 Mendelson BC. Surgery of the superficial musculoaponeurotic system: principles of release, vectors, and fixation. Plast Reconstr Surg 2001;107(06):1545-1552, discussion 1553-1555, 15561557, 1558-1561

12 Beaty MM. Imbrication, plication and wide undermining techniques. In: Gentile RD, ed. Neck Rejuvenation. New York, NY: Thieme; 2011

13 Hamra ST. The deep-plane rhytidectomy. Plast Reconstr Surg 1990;86(01):53-61, discussion 62-63

14 Jacono AA, Parikh SS. The minimal access deep plane extended vertical facelift. Aesthetic Surg J 2011;31(08):874-890

15 Gentile RD. Mixed-plane rhytidectomy: the superior verticalvector approach to rejuvenation of the neck. In: Gentile RD, ed. Neck Rejuvenation. New York, NY: Thieme; 2011

16 Gordon NA, Sawan TG. Deep plane approach to the vertical platysma advancement: technical modifications and nuances over 25 years. Facial Plast Surg 2020;36(04):358-375

17 Beaty MM. A progressive approach to neck rejuvenation. Facial Plast Surg Clin North Am 2014;22(02):177-190

18 Hamra ST. Composite rhytidectomy. Plast Reconstr Surg 1992;90 (01):1-13

19 Bassichis BA. Volumetric facelift with intra- and post-operative midface volume replacement "the four-dimensional facelift". Facial Plast Surg Clin North Am 2009;17(04):539-547, v-vi

20 Coleman SR. Long-term survival of fat transplants: controlled demonstrations. Aesthetic Plast Surg 1995;19(05): 421-425 